\title{
Anthocyanin and Isoflavone Contents in Korean Black Soybean Landraces and Their Antioxidant Activities
}

\author{
Kyung Jun Lee ${ }^{1}$, Jung-Ro Lee ${ }^{1}$, Kyung-Ho Ma $^{1}$, Yang-Hee Cho ${ }^{1}$, Gi-An Lee ${ }^{1}$, Jong-Wook Chung ${ }^{2}$ * \\ ${ }^{1}$ National Agrobiodiversity Center, Rural Development Administration, Jeonju 54874, Korea \\ ${ }^{2}$ Department of Industrial Plant Science and Technology, Chungbuk National University, Cheongju 28644, Korea
}

\begin{abstract}
Anthocyanin and isoflavone contents and antioxidant activities of 56 Korean black soybean landraces were examined in this study. Total isoflavone content of 56 Korean black soybean landraces ranged from 43.8 to $347.5 \mathrm{mg} / 100 \mathrm{~g}$. Total anthocyanin content ranged from 19.8 to $1,420.4 \mathrm{mg} / 100 \mathrm{~g}$, with cyaniding-3-glucoside being the most abundant anthocyanin in all landraces, ranging from 12.2 to $1,207.3 \mathrm{mg} / 100 \mathrm{~g}$. Antioxidant activities based on 2,2-diphenyl-1-picrylhydrazyl, 2,2'-azino-bis(3ethylbenzothiazoline-6-sulphonic acid), ferric-reducing antioxidant power, and total polyphenol content assays showed wide variations, ranging from 17.1 to 76.6 (IC50), 53.5 to 127.7 (IC50), 5.4 to $34.2 \mathrm{mg}$ ascorbic acid/g, and 9.7 to $36.8 \mathrm{mg}$ gallic acid equivalents/g), respectively. Using relative antioxidant capacity index, IT156132 sample had the highest antioxidant activity. In clustering analysis, the 56 Korean black soybean landraces were classified into five groups. Group I consisted of four landraces with high isoflavone contents and antioxidant activities.
\end{abstract}

Keywords Antioxidant activity, Anthocyanin, Korean black soybean landraces, Isoflavone

\section{INTRODUCTION}

Various kinds of soybeans (Glycine max L. Merr.) are cultivated in East Asia as diverse food sources after adapting in different environments for a long time (Cho et al. 2008). Previous studies have reported that Korean soybean landraces have large genetic variations (Kwon et al. 1974; Perry and Mclntosh 1991; Song et al. 1991; Yoon 2003). Many countries such as America, Canada, China, and Japan have developed various soybean cultivars with traits such as high yield, disease resistance, and tolerance of environment stress using Korean soybean landraces (Lee 2003).

Soybean is a well-known food staple that contains starch, dietary fiber, protein, lipids, and essential minerals as well as beneficial secondary metabolites (Bellaloui 2012). Recently, black soybeans have been found to contain high contents of $\gamma$-tocopherol, isoflavones, flavonoids, and anthocyanins with biological activity (Correa et al. 2010). Antioxidant properties resulting from free radical scavenging effects and total phenolic compounds have been found to be higher in black soybeans compared to yellow soybeans (Dajanta et al. 2013).

Anthocyanins are secondary metabolites and watersoluble pigments that are responsible for the red, purple, or blue coloration of many fruits, vegetables, and cereal grains (Kim et al. 2012). They play various important roles in plants, such as attracting animals participating in pollination and seed disposal, repelling harmful insects, and protecting plants against damages caused by ultraviolet light (Shin et al. 2009). The black color in soybean is ascribed to an accumulation of anthocyanins in its epidermis palisade layer (Todd and Vodkin 1993). Three main anthocyanins (delphinidin-3-O- $\beta$-D-glucoside $[\mathrm{D} 3 \mathrm{G}]$, cyanidin 3-O- $\beta$-D-glucoside [C3G], and petunidin-3-O- 
$\beta$-D-glucoside [Pt3G]) have been detected in the seed coat of black soybean (Choung et al. 2001).

Consumption of isoflavones is associated with benefits to human health, such as decreased risk of heart disease, reduced menopausal symptoms, and reduced risk of some hormone-related cancers (Yao et al. 2004). Isoflavones in soybeans are mainly aglycones (i.e., daidzein, genistein, glycitein), $\beta$-glucosides (i.e., daidzin, genistin, glycitin), malonyl- $\beta$-glucosides (i.e., 6"-O-malonyldaidzin, 6"-Omalonylgenistin, 6"-O-malonylglycitin), and acetyl- $\beta$ glucosides (i.e., 6"-O-acetyldaidzin, 6"-O-acetylgenistin, 6"-O-acetylglycitin) (Lee and Lee 2009). Aglycones are flavonoid molecules lacking any attached sugars or other modifiers. Among different forms of isoflavones, aglycones are especially important because they are readily bioavailable to humans (Lee and Lee 2009).

Antioxidant compounds have received attention from natural-product consumers and researchers due to their pharmacological properties. Antioxidants can lower oxidative stress caused by reactive oxygen species (Nordberg and Arnér 2001). There is an increasing interest in natural antioxidant products for use as medicines and food additives (Mossi et al. 2004; Willcox et al. 2004). Phytochemicals such as polyphenols and carotenoids are important because they contribute to human health with multiple biological effects such as antioxidant, antimutagenic, anticarcinogenic, and cytoprotective activities (Ajila and Prasada Rao 2008).

In this study, 56 Korean black soybean landraces were analyzed to determine anthocyanins and isoflavones contents and antioxidant activities. In addition, the relation among anthocyanins, isoflavones, and antioxidant activity of samples was tested. Furthermore, black soybean landraces with high anthocyanin and isoflavone contents and antioxidant activities were identified.

\section{MATERIALS AND METHODS}

\section{Plant materials}

Fifty-six Korean black soybean lnadraces were obtained from the National Agrobiodiversity Center of the Rural Development Administration, Korea (http://genebank.rda. go.kr).

\section{Analysis of anthocyanins in soybean seeds}

One-hundred mg of each Korean black soybean landrace was mixed with $15 \mathrm{ml}$ of $1 \% \mathrm{HCl}$ in $99 \% \mathrm{MeOH}$ for 24 hours at $4^{\circ} \mathrm{C}$ in the dark. After centrifugation at 13,000 rpm for 10 minutes, each specimen was filtered through a 0.45 $\mu \mathrm{m}$ syringe filter and analyzed with Agilent 1260 Infinity HPLC system (Agilent Technol., Santa Clara, CA, USA). The analysis was performed using Waters XSelect HSS Cyano XP column $(2.5 \mu \mathrm{m}, 2.1 \times 75 \mathrm{~mm}$; Waters, Milford, MA, USA). HPLC conditions were as follows: solvent A, $0.1 \% \mathrm{TFA} / \mathrm{H}_{2} \mathrm{O}$; solvent $\mathrm{B}, 0.1 \% \mathrm{TFA} / \mathrm{CH}_{3} \mathrm{CN}$; gradient, $5 \%$ (B) for 0.3 minutes, $20 \%$ (B) for 6.0 minutes, $95 \%$ (B) for 8.0 minutes, and $5 \%$ (B) for 10 minutes; column temperature, $40^{\circ} \mathrm{C}$; and flow rate, $0.5 \mathrm{ml} / \mathrm{min}$. The filter detector was set at $520 \mathrm{~nm}$.

\section{Analysis of isoflavones in soybean seeds}

One-hundred milligram of each sample was added to 2 $\mathrm{ml}$ of $80 \% \mathrm{MeOH}$ and sonicated for 1 hour. The sample in each tube was hydrolyzed using $150 \mu 1$ of $2 \mathrm{~N} \mathrm{NaOH}$. After mixing for 10 minutes, the solution was neutralized with 50 $\mu 1$ of glacial acetic acid. The sample was centrifuged at $3,000 \mathrm{rpm}$ for 5 minutes. Supernatant was collected and filtered using a $0.45 \mu \mathrm{m}$ syringe filter prior to analysis by with Agilent 1260 Infinity HPLC system (Agilent Technol.). The analysis was performed using a Proshell 120 SB-C15 column $(2.7 \mu \mathrm{m}, 2.1 \times 50 \mathrm{~mm}$; Agilent Technol.). HPLC conditions were as follows: solvent $\mathrm{A}, 0.1 \% \mathrm{TFA} / \mathrm{H}_{2} \mathrm{O}$; solvent $\mathrm{B}, 0.1 \% \mathrm{TFA} / \mathrm{CH}_{3} \mathrm{CN}$; gradient, $10 \%$ (B) for 0.35 minutes, $10 \%$ to $30 \%$ (B) in 3.96 minutes, hold at $30 \%$ (B) for 0.36 minutes, re-equilibrate at $10 \%$ (B) for 1.8 minutes; column temperature, $30^{\circ} \mathrm{C}$; and flow rate, $0.58 \mathrm{ml} / \mathrm{min}$. The filter detector was set at $254 \mathrm{~nm}$.

\section{Extraction for antioxidant activities}

One-hundred milligram of each soybean powder was added to $1 \mathrm{ml}$ of $80 \% \mathrm{EtOH}$. The mixture was sonicated in an ultrasonic bath for 3 hours at room temperature. The suspension was centrifuged at 13,000 rpm for 10 minutes. The clear supernatant was then used for 2,2-diphenyl1-picrylhydrazyl (DPPH), 2,2'-azino-bis(3-ethylbenzo- 
thiazoline-6-sulphonic acid) (ABTS), ferric-reducing antioxidant power (FRAP), and total polyphenol content (TPC) assays.

\section{DPPH assay}

DPPH radical-scavenging activities of extracts were assessed using published method with slight modifications (Lee and Lee 2004). Briefly, DPPH solution (150 $\mu$; 150 $\mu \mathrm{M}$ in anhydrous EtOH) was added to $100 \mu \mathrm{l}$ of sample solution. The mixture was shaken vigorously and left to stand at $25^{\circ} \mathrm{C}$ in the dark for 30 minutes. Absorbance at 517 $\mathrm{nm}$ was measured using a spectrophotometer (Epoch; Bio-Tek, Winooski, VT, USA). Results were expressed as IC50 and compared to ascorbic acid standard.

\section{ABTS assay}

ABTS radical-scavenging activity was estimated using previously described method with modifications (Re et al. 1999). Briefly, ABTS radical cation was generated by adding $7 \mathrm{mM}$ ABTS to $2.45 \mathrm{mM}$ potassium persulfate followed by an overnight incubation in the dark at room temperature. The ABTS radical cation solution was diluted with methanol $(\mathrm{MeOH})$ to obtain an absorbance of $0.7 \pm 0.02$ at $735 \mathrm{~nm}$. The diluted ABTS radical cation solution $(190 \mu \mathrm{l})$ was added to $10 \mu \mathrm{l}$ of sample solution. After 6 minutes of incubation, absorbance at $735 \mathrm{~nm}$ was determined using a spectrophotometer. Results were expressed as IC50 and compared to ascorbic acid standard.

\section{FRAP}

The reducing power of the 56 Korean black soybean landraces was determined using published method with slight modifications (Yen and Duh 1993). Briefly, $0.1 \mathrm{ml}$ aliquot of the extract was mixed with a $0.5 \mathrm{ml}$ phosphate buffer $(0.2 \mathrm{M}, \mathrm{pH} 6.6)$ containing $1 \% \mathrm{~K} 3 \mathrm{Fe}(\mathrm{CN}) 6$. The mixture was incubated at $50^{\circ} \mathrm{C}$ for 20 minutes. After centrifugation at $200 \mathrm{~g}$ for 10 minutes, the upper layer $(10 \mu \mathrm{l})$ was mixed with $390 \mu 1$ of $1 \%$ ferric chloride. The absorbance was monitored at $700 \mathrm{~nm}$ using a spectrophotometer.

\section{TPC assay}

TPC was measured using modified Folin-Ciocalteu method (Waterhouse 2003). Folin-Ciocalteu reagent (100 $\mu \mathrm{l})$ was added to $100 \mu \mathrm{l}$ of sample solution and reacted at room temperature for 3 minutes. After adding $100 \mu \mathrm{l}$ of $2 \%$ sodium carbonate, the mixture was incubated at room temperature for 30 minutes. Absorbance was measured at $750 \mathrm{~nm}$ on a spectrophotometer using distilled water as the blank. Total phenolic content was reported as milligrams of gallic acid equivalents (GAE) per gram of dry weight sample (mg GAE $\mathrm{mg}^{-1}$ dry seed).

\section{Data analysis}

Duncan's multiple-range test and correlation analysis were used to determine differences among the 56 Korean black soybean landraces using IBM SPSS Statistics ver. 20 (IBM Co., Armonk, NY, USA). Cluster analysis was performed using $\mathrm{R}$ statistical software environment (http://www.r-project.org). Software PAST3 was used for principal component analyses (PCA) (Hammer et al. 2001). Statistical significance was considered when $P$-value was less than 0.05 .

\section{RESULTS}

\section{Isoflavone content}

Total and individual isoflavone contents of the 56 Korean black soybean landraces are summarized in Table 1 and Supplementary Table 1. Composite values for six isoflavones (namely daidzin, genistin, glycitin, daidzein, genistein, and glycitein) were analyzed and expressed as total isoflavone content. Total isoflavone contents (TIC) of Korean black soybean landraces ranged from 43.8 to 347.5 $\mathrm{mg} / 100 \mathrm{~g}$. Among soybean landraces, sample IT177372 had the highest TIC content $(347.5 \pm 2.5 \mathrm{mg} / 100 \mathrm{~g})$. The content of isoflavone glycosies ranged from 39.1 to 335.5 $\mathrm{mg} / 100 \mathrm{~g}$. Among isoflavone glycosides, daidzin, glycitin, and genistin contents ranged from 12.3 to $153.5,2.4$ to 35.5 , and 20.8 to $146.6 \mathrm{mg} / 100 \mathrm{~g}$, respectively, while daidzein, glycitein and genistein contents ranged from 0.3 to $9.2,0.7$ to 14.9 , and 0.2 to $3.1 \mathrm{mg} / 100 \mathrm{~g}$, respectively.

\section{Anthocyanin content}

The following three anthocyanins, D3G, C3G, and Pt3G, were detected in 56 Korean black soybean landraces 
Table 1. Descriptive statistics of anthocyanins, isoflavones, and antioxidant activities of the 56 Korean black soybean landraces used in this study.

\begin{tabular}{clcccc}
\hline \hline Variable & \multicolumn{1}{c}{ Subtotal } & $\begin{array}{c}\text { Range } \\
\text { (minimum-maximum) }\end{array}$ & $\begin{array}{c}\text { Mean } \pm \text { standard } \\
\text { deviation }\end{array}$ & Skewness & Kurtosis \\
\hline Isoflavone content & Daidzin & $12.3-153.5$ & $55.5 \pm 23.3$ & 1.37 & 5.08 \\
$(\mathrm{mg} / 100 \mathrm{~g})$ & Glycitin & $2.4-35.5$ & $16.4 \pm 7.3$ & 0.62 & 0.81 \\
& Genistin & $20.8-146.6$ & $70.8 \pm 26.0$ & 0.37 & 0.38 \\
& Glycosides & $39.1-335.5$ & $142.7 \pm 51.9$ & 0.69 & 2.82 \\
& Daidzein & $0.3-9.2$ & $2.6 \pm 2.0$ & 1.43 & 2.30 \\
& Glycitein & $0.7-14.9$ & $3.5 \pm 2.9$ & 2.70 & 7.65 \\
& Genistein & $0.2-3.1$ & $1.2 \pm 0.8$ & 1.12 & 0.37 \\
Anthocyanin content & $1.7-18.8$ & $7.3 \pm 3.8$ & 1.19 & 1.55 \\
(mg/100 g) & Aglycones & $43.8-347.5$ & $149.9 \pm 53.7$ & 0.61 & 2.70 \\
& DIC & $0.0-216.4$ & $63.9 \pm 44.8$ & 1.22 & 2.14 \\
& C3G & $12.2-1207.3$ & $333.6 \pm 295.6$ & 1.19 & 0.84 \\
Antioxidant activity & Pt3G & $0.0-42.8$ & $12.0 \pm 10.0$ & 1.39 & 1.61 \\
& TAC & $19.8-1420.4$ & $433.5 \pm 335.2$ & 1.08 & 0.71 \\
& DPPH (IC50) & $17.1-76.6$ & $59.3 \pm 15.6$ & -1.18 & 0.62 \\
& ABTS (IC50) & $53.5-127.7$ & $75.1 \pm 20.3$ & 1.17 & 0.57 \\
& FRAP (mg ASC/g) & $5.4-34.2$ & $15.5 \pm 6.9$ & 0.48 & -0.44 \\
& TPC (mg GAE/g) & $9.7-36.8$ & $20.0 \pm 6.5$ & 0.48 & -0.47 \\
\hline
\end{tabular}

${ }^{2)}$ TIC: total isoflavone content, D3G: delphinidin-3-O- $\beta$-D-glucoside, C3G: cyanidin 3-O- $\beta$-D-glucoside, Pt3G: petunidin3-O- $\beta$-D-glucoside, TAC: total anthocyanin content, DPPH: 2,2-diphenyl-1-picrylhydrazyl, ABTS: 2,2'-azino-bis(3ethylbenzothiazoline-6-sulphonic acid), FRAP: ferric-reducing antioxidant power, ASC: ascorbic acid, TPC: total polyphenol content, GAE: gallic acid equivalents.

(Table 1 and Supplementary Table 1). Total anthocyanin content (TAC) ranged from 19.8 to $1,420.4 \mathrm{mg} / 100 \mathrm{~g}$. Among soybean landraces, sample IT177257 had the highest TAC content $(1,420.4 \pm 1.8 \mathrm{mg} / \mathrm{g})$, while sample IT112847 had the lowest TAC content $(19.8 \pm 2.1 \mathrm{mg} / \mathrm{g})$. $\mathrm{C} 3 \mathrm{G}$ was the main component in anthocyanin (ranging from 12.2 to $1,207.3 \mathrm{mg} / 100 \mathrm{~g}$ ). D3G and Pt3G contents ranged from 0.0 to $216.4 \mathrm{mg} / 100 \mathrm{~g}$ and 0.0 to $42.8 \mathrm{mg} / 100$ $\mathrm{g}$, respectively.

\section{Antioxidant activity}

DPPH, ABTS, FRAP, and TPC were used to determine the antioxidant activities of the 56 Korean black soybean landraces. Results are summarized in Table 1 and Supplementary Table 1. DPPH radical-scavenging activities of soybean landraces ranged from 17.1 to 76.6 (IC50). Sample IT156132 had the highest DPPH radical-scavenging activity (17.1 \pm 8.2 , IC50). The ABTS antioxidant activities of soybean landraces ranged from 53.5 to 127.7 (IC50). Sample IT177485 and IT177530 had the highest ABTS- scavenging activity (53.5 \pm 0.1 , IC50). FRAP of soybean landraces ranged from 5.4 to $34.2 \mathrm{mg}$ ascorbic acid (ASC)/g. Sample IT177253 had the highest FRAP (34.2 $\pm 1.5 \mathrm{mg}$ ASC/g) while IT177430 had the lowest FRAP $(5.4 \pm 0.2 \mathrm{mg} \mathrm{ASC} / \mathrm{g})$. TPC of soybean landraces ranged from 9.7 to $36.8 \mathrm{mg} \mathrm{GAE} / \mathrm{g}$. Sample IT177370 had the highest TPC ( $36.8 \pm 3.4 \mathrm{mg} \mathrm{GAE} / \mathrm{g})$ while IT177430 had the lowest TPC $(9.7 \pm 0.2 \mathrm{mg} \mathrm{GAE} / \mathrm{g})$. The integration of antioxidant capacity results derived from different chemical methods allowed us to calculate the relative antioxidant capacity index (RACI). Results are shown in Fig. 1. It was found that IT156132 had the highest RACI (2.12), followed by IT177485 (1.37), IT177370 (1.34), and IT177253 (1.31), with IT177430 having lowest value of RACI $(-1.30)$.

\section{Correlation analysis}

The correlations among anthocyanins, isoflavones, and antioxidant activities were analyzed. Results are shown in Table 2. There was no significant correlation 


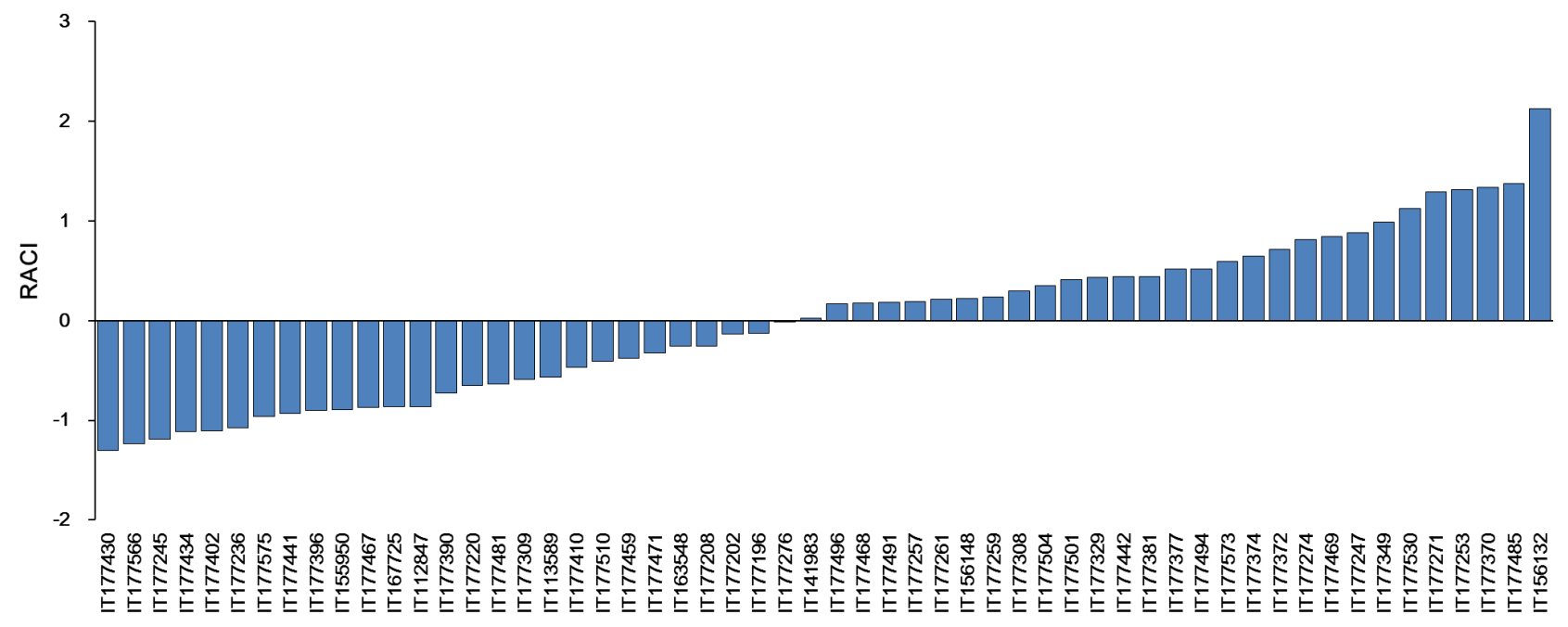

Fig. 1. Relative antioxidant capacity index (RACI) of 56 Korean black soybean landraces.

Table 2. Correlations among anothocyanins, isoflavones, and antioxidant activities of the 56 Korean black soybean landraces.

\begin{tabular}{|c|c|c|c|c|c|c|c|c|c|c|c|c|c|c|c|c|c|}
\hline Variable & $\mathrm{DPPH}^{\mathrm{z}) \mathrm{y})}$ & $\mathrm{ABTS}^{\mathrm{z})}$ & FRAP & TPC & Daidzin & Glycitin & Genistin & Glycosides & Daidzein & Glycitein & Genistein & Aglycones & $\begin{array}{l}\text { TIC } \\
\end{array}$ & D3G & C3G & Pt3G & TAC \\
\hline ABTS & 0.127 & & & & & & & & & & & & & & & & \\
\hline FRAP & -0.241 & $-0.792^{* *}$ & & & & & & & & & & & & & & & \\
\hline TPC & $-0.294^{*}$ & $-0.727^{* *}$ & $0.838^{* *}$ & & & & & & & & & & & & & & \\
\hline Daidzin & -0.089 & 0.070 & 0.089 & 0.078 & & & & & & & & & & & & & \\
\hline Glycitin & -0.111 & 0.120 & 0.071 & 0.049 & $0.872^{* *}$ & & & & & & & & & & & & \\
\hline Genistin & -0.121 & 0.033 & 0.024 & -0.078 & $0.738^{* *}$ & $0.594 * *$ & & & & & & & & & & & \\
\hline Glycosides & -0.116 & 0.065 & 0.062 & 0.003 & $0.942^{* *}$ & $0.831^{* *}$ & $0.917^{* *}$ & & & & & & & & & & \\
\hline Daidzein & -0.007 & 0.111 & 0.038 & -0.074 & $0.466^{* *}$ & $0.418^{* *}$ & $0.625^{* *}$ & $0.582^{* *}$ & & & & & & & & & \\
\hline Glycitein & 0.080 & 0.039 & -0.208 & -0.156 & 0.146 & -0.006 & 0.130 & 0.130 & -0.112 & & & & & & & & \\
\hline Genistein & 0.082 & -0.117 & -0.050 & 0.084 & 0.185 & 0.004 & $0.330^{*}$ & 0.249 & -0.006 & $0.564^{* *}$ & & & & & & & \\
\hline Aglycones & 0.077 & 0.067 & -0.155 & -0.142 & $0.391^{* *}$ & 0.211 & $0.490^{* *}$ & $0.451^{* *}$ & $0.425^{* *}$ & $0.834^{* *}$ & $0.646^{* *}$ & & & & & & \\
\hline TIC & -0.107 & 0.068 & 0.049 & -0.007 & $0.938^{* *}$ & $0.817^{* *}$ & $0.920^{* *}$ & $0.998^{* *}$ & $0.592^{* *}$ & 0.185 & $0.287 *$ & $0.507^{* *}$ & & & & & \\
\hline D3G & $-0.267^{*}$ & -0.105 & 0.175 & 0.176 & 0.142 & 0.121 & 0.100 & 0.131 & -0.032 & -0.198 & -0.131 & -0.199 & 0.113 & & & & \\
\hline $\mathrm{C} 3 \mathrm{G}$ & -0.076 & -0.053 & 0.052 & 0.057 & 0.207 & 0.256 & 0.194 & 0.226 & -0.095 & -0.092 & -0.024 & -0.127 & 0.210 & $0.314^{*}$ & & & \\
\hline Pt3G & $-0.266^{*}$ & -0.023 & 0.109 & 0.059 & 0.162 & 0.188 & 0.129 & 0.164 & 0.087 & -0.234 & -0.258 & -0.194 & 0.145 & $0.752^{* *}$ & -0.003 & & \\
\hline TAC & $-0.289^{*}$ & -0.207 & 0.261 & 0.234 & 0.188 & 0.173 & 0.153 & 0.185 & -0.001 & -0.223 & -0.159 & -0.210 & 0.164 & $0.835^{* *}$ & $0.361^{* *}$ & $0.665^{* *}$ & \\
\hline RACI & $-0.502^{* *}$ & $-0.830^{* *}$ & $0.916^{* *}$ & $0.891 * *$ & 0.051 & 0.013 & 0.030 & 0.040 & -0.020 & -0.133 & 0.035 & -0.109 & 0.031 & 0.214 & 0.022 & 0.162 & $0.296^{*}$ \\
\hline
\end{tabular}

$* P<0.05, * * P<0.01$.

${ }^{z)} \mathrm{DPPH}$ and ABTS: IC50.

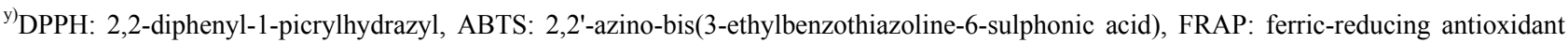
power, TPC: total polyphenol content, TIC: total isoflavone content, D3G: delphinidin-3-O- $\beta$-D-glucoside, C3G: cyanidin 3-O- $\beta$-D-glucoside, Pt3G: petunidin-3-O- $\beta$-D-glucoside, TAC: total anthocyanin content, RACI: relative antioxidant capacity index.

between anthocyanins and isoflavones or between antioxidant activities and isoflavones. DPPH (IC50) showed negative correlations with TPC $(\mathrm{r}=-0.294, P$ $<0.05)$, D3G $(\mathrm{r}=-0.267, P<0.05), \mathrm{Pt3G}(\mathrm{r}=-0.266, P$ $<0.05)$, and TAC $(\mathrm{r}=-0.289, P<0.05)$. ABTS (IC50) only showed negative correlation with the following two antioxidant activities: FRAP (r=-0.792, $P<0.01)$ and TPC $(\mathrm{r}=-0.727, P<0.01)$. FRAP showed a positive correlation with TPC ( $\mathrm{r}=0.838, P<0.01)$. RACI showed negative correlations with DPPH $(\mathrm{r}=-0.502, P<0.01)$ and ABTS $(\mathrm{r}=-0.830, P<0.01)$. However, it had positive correlations with FRAP $(\mathrm{r}=0.916, \quad P<0.01)$, TPC $(\mathrm{r}=0.891, P<0.01)$, and TAC $(\mathrm{r}=0.296, P<0.05)$.

\section{Principal component analyses (PCA) analysis}

PCA analysis was performed for anthocyanins iso- 
flavones. Results of PCA analysis are shown in Table 3. The cumulative variance of the first five axes with Eigen value of $>1.0$ was $84.0 \%$. The first PC was more related to isoflavone glycosides, daidzin, glycitin, and genistin than TIC. In second principal component, the antioxidant activities (ABTS, FRAP, TPC) and RACI were more related traits except DPPH. The third principal component exhibited positive effects on isoflavones (aglycones, daidzein, glycitein, and genistein) and ABTS. However, it had negative effects on Pt3G and TAC. The fourth principal component was also more related to isoflavones (aglycones, daidzein, glycitein, and genistein) and TAC. The fifth principal component exhibited positive effect on C3G but negative effect on daidzein, Pt3G, and DPPH. The distribution of Korean black soybean landraces in PCA analysis is shown in Fig. 2. After placing an ellipse around the data with $95 \%$ confidence interval using Hotelling's T2 statistic, it was possible to observe all soybean landraces except two (IT177485 and IT177372). IT177485 showed the lowest content of isoflavone aglycones (daidzein, $0.3 \pm 0.0 \mathrm{mg} / 100 \mathrm{~g}$; glycitein, $0.7 \pm 0.1 \mathrm{mg} / 100 \mathrm{~g}$; total aglycones, $1.7 \pm 0.2 \mathrm{mg} / 100 \mathrm{~g}$ ), while IT177372 had the highest levels of isoflavone glycosides (daidzin, 153.5 \pm 1.4 $\mathrm{mg} / 100 \mathrm{~g}$; glycitein, $35.4 \pm 0.3 \mathrm{mg} / 100 \mathrm{~g}$; genistein, $146.6 \pm$ $0.2 \mathrm{mg} / 100 \mathrm{~g}$; total glycoside, $335.5 \pm 1.6 \mathrm{mg} / 100 \mathrm{~g})$.

\section{Clustering analysis}

The 56 Korean black soybean landraces were classified into five clusters according to their anthocyanins, isoflavones, and antioxidant activities (Table 4 and Fig. 3). There was no significant difference in D3G, Pt3G, or DPPH among the five clusters. Cluster I contained four landraces (IT177372, IT177349, IT177253, and IT177309). They had high contents of daidzin, glycitin, genistin, total isoflavone glycosides, TIC, and C3G. Cluster II contained seven landraces with high glycitein and total isoflavone

Table 3. Eigen value, percent of total variation, and component matrix for the principal component axes.

\begin{tabular}{lcccrr}
\hline \hline Principal components & $\mathrm{PC}^{\mathrm{z}} 1$ & PC2 & PC3 & PC4 & PC5 \\
\hline Eigen value & 5.35 & 4.22 & 2.71 & 1.60 & 1.24 \\
\% of variance & 29.7 & 23.5 & 15.1 & 8.9 & 6.9 \\
Cumulative \% & 29.7 & 53.2 & 68.3 & 77.1 & 84.0 \\
Component matrix & & & & \\
Daidzin & 0.398 & -0.010 & -0.031 & -0.109 & 0.094 \\
Glycitin & 0.351 & 0.001 & -0.115 & -0.213 & 0.111 \\
Genistin & 0.390 & -0.042 & 0.011 & -0.032 & -0.050 \\
Glycosides & 0.424 & -0.026 & -0.024 & -0.095 & 0.033 \\
Daidzein & 0.268 & -0.054 & -0.009 & -0.267 & -0.374 \\
Glycitein & 0.090 & -0.204 & 0.315 & 0.475 & 0.063 \\
Genistein & 0.132 & -0.114 & 0.354 & 0.356 & 0.174 \\
Aglycones & 0.234 & -0.211 & 0.314 & 0.305 & -0.107 \\
TIC & 0.426 & -0.040 & -0.001 & -0.070 & 0.024 \\
D3G & 0.089 & 0.261 & -0.339 & 0.383 & 0.047 \\
C3G & 0.100 & 0.081 & -0.174 & 0.038 & 0.702 \\
Pt3G & 0.096 & 0.218 & -0.355 & 0.285 & -0.315 \\
TAC & 0.110 & 0.290 & -0.309 & 0.315 & 0.090 \\
DPPH & 0.094 & 0.217 & 0.049 & 0.219 & -0.408 \\
ABTS & -0.017 & 0.370 & 0.309 & -0.044 & 0.066 \\
FRAP & 0.045 & 0.408 & 0.238 & -0.160 & 0.035 \\
TPC & 0.024 & 0.390 & 0.263 & -0.084 & 0.104 \\
RACI & 0.045 & 0.430 & 0.266 & -0.021 & -0.063 \\
\hline
\end{tabular}

${ }^{\mathrm{z})} \mathrm{PC}$ : principal component, TIC: total isoflavone content, D3G: delphinidin-3-O- $\beta$-D-glucoside, C3G: cyanidin 3-O- $\beta$-D-glucoside, Pt3G: petunidin-3-O- $\beta$-D-glucoside, TAC: total anthocyanin content, DPPH: 2,2-diphenyl-1picrylhydrazyl, ABTS: 2,2'-azino-bis(3-ethylbenzothiazoline-6-sulphonic acid), FRAP: ferric-reducing antioxidant power, TPC: total polyphenol content, RACI: relative antioxidant capacity index. 


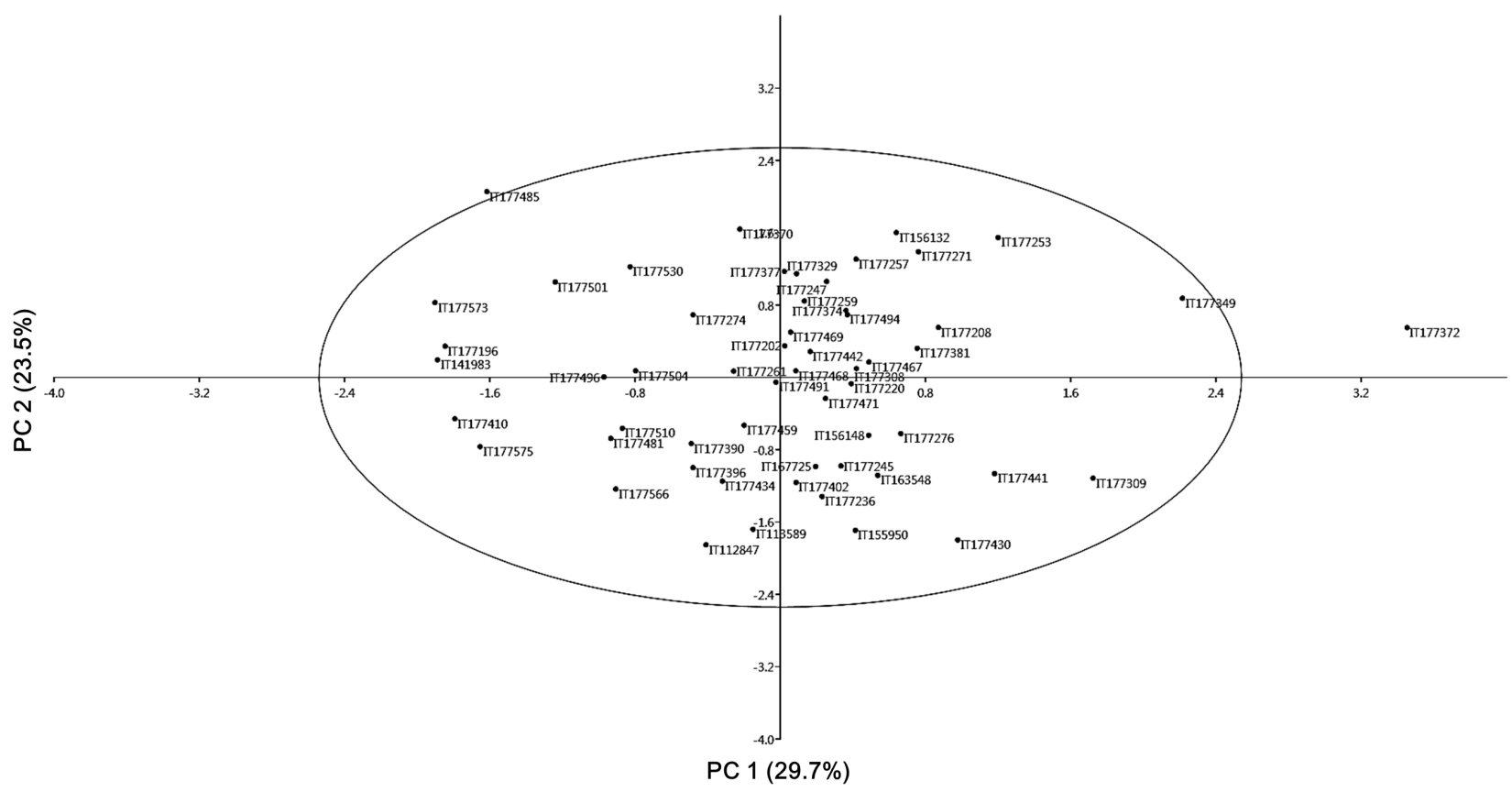

Fig. 2. Two-dimensional scatter diagram of principal component analysis of 56 Korean black soybean landraces based on anthocyanins, isoflavones, and antioxidant activities.

aglycones but low $\mathrm{C} 3 \mathrm{G}$ and TAC. Cluster III and IV consisted of 13 and 22 soybean landraces, respectively. They had high TAC. Cluster V comprised 10 landraces with low daidzin, glycitin, genistin, total isoflavon glycosides, daidzein, total isoflavone aglycones, and TIC. In antioxidant activities, Cluster III showed low ABTS while cluster IV had high ABTS. In FRAP, TPC, and RACI, Cluster I, III, and V showed higher antioxidant activities compared to Cluster II or IV.

\section{DISCUSSION}

Black soybean (Glycine max L.) has been widely utilized as a health food and herbal material in Oriental medicine for hundreds of years (Xu and Chang 2008a). For centuries, farming communities have continuously contributed to the evolution, enrichment, and maintenance of landrace diversity on-farm (Brush 1995; Jarvis et al. 2008). However, little has been done to understand the landrace diversity or to improve these landraces (Sthapit and Rao 2009). Sthapit and Rao (2009) have suggested that landraces can be effectively improved by simple trait selection if these landraces could offer sufficient natural variations in the population. Our results revealed that the 56 Korean black soybean landraces had different compositions in anthocyanin and isoflavone (Table 1).

Antioxidant capacities of plant extracts not only depend on extract composition, but also depend on the conditions of test used (Dudonné et al. 2009). In this study, we determined free radical scavenging capacities of Korean black soybean landraces using DPPH, ABTS, FRAP, or TPC assay. These methods have been widely used to determine the antioxidant capacities of plant extracts as they require relatively standard equipment. In addition, they can deliver fast and reproducible results (Dudonné et al. 2009). Sun and Tanumihardjo (Sun and Tanumihardjo 2007) have proposed a RACI because each method to measure the antioxidant capacity has its own limitations. Multiple reaction mechanisms and different phase locations are usually involved in the measurement of antioxidant capacity (Xu and Chang 2008a). The key advantage of RACI is that it is a numerical scale that integrates multiple chemical methods, thus allowing comparison of antioxidant capacity for a large number of samples (Sun and Tanumihardjo 2007). Our results showed that the 56 
Table 4. Average values \pm standard deviation of each cluster based on anthocyanins, isoflavones, and antioxidant activities.

\begin{tabular}{|c|c|c|c|c|c|}
\hline \multirow{2}{*}{ Variable } & \multicolumn{5}{|c|}{ Cluster } \\
\hline & I $(n=4)$ & II $(n=7)$ & III $(n=13)$ & IV $(n=22)$ & $V(n=10)$ \\
\hline \multicolumn{6}{|c|}{ Isoflavone $(\mathrm{mg} / 100 \mathrm{~g})$} \\
\hline Daidzin & $112.7 \pm 29.1 \mathrm{c}$ & $57.4 \pm 7.2 b$ & $57.4 \pm 11.3 b$ & $56.2 \pm 12.5 b$ & $27.4 \pm 11.5 \mathrm{a}$ \\
\hline Glycitin & $32.9 \pm 4.2 \mathrm{~d}$ & $13.5 \pm 2.6 b$ & $17.9 \pm 3.9 \mathrm{c}$ & $17.9 \pm 4.4 \mathrm{c}$ & $6.5 \pm 2.8 \mathrm{a}$ \\
\hline Genistin & $112.6 \pm 23.5 \mathrm{c}$ & $82.9 \pm 21.0 \mathrm{~b}$ & $70.5 \pm 12.9 b$ & $74.6 \pm 20.9 b$ & $37.7 \pm 16.0 \mathrm{a}$ \\
\hline Glycosides & $258.1 \pm 55.2 \mathrm{c}$ & $153.8 \pm 29.2 b$ & $145.7 \pm 15.6 b$ & $148.6 \pm 29.3 b$ & $71.6 \pm 28.1 \mathrm{a}$ \\
\hline Daidzein & $4.3 \pm 1.9 b$ & $3.6 \pm 3.6 b$ & $3.1 \pm 1.6 \mathrm{~b}$ & $2.5 \pm 1.2 \mathrm{~b}$ & $0.6 \pm 0.3 \mathrm{a}$ \\
\hline Glycitein & $4.0 \pm 0.5 \mathrm{a}$ & $9.1 \pm 5.1 b$ & $3.2 \pm 1.4 \mathrm{a}$ & $2.6 \pm 0.8 \mathrm{a}$ & $1.9 \pm 1.4 \mathrm{a}$ \\
\hline Genistein & $1.2 \pm 1.0 \mathrm{a}$ & $2.1 \pm 0.9 \mathrm{~b}$ & $1.4 \pm 1.0 \mathrm{a}$ & $0.9 \pm 0.5 \mathrm{a}$ & $0.8 \pm 0.4 \mathrm{a}$ \\
\hline Aglycones & $9.5 \pm 2.2 \mathrm{c}$ & $14.7 \pm 3.0 \mathrm{~d}$ & $7.7 \pm 2.4 \mathrm{bc}$ & $6.0 \pm 1.1 \mathrm{~b}$ & $3.3 \pm 1.7 \mathrm{a}$ \\
\hline $\mathrm{TIC}^{\mathrm{z})}$ & $267.6 \pm 57.2 \mathrm{c}$ & $168.6 \pm 26.9 b$ & $153.4 \pm 16.5 b$ & $154.7 \pm 29.8 b$ & $74.8 \pm 29.2 \mathrm{a}$ \\
\hline \multicolumn{6}{|c|}{ Anthocyanin (mg/100 g) } \\
\hline D3G & $73.7 \pm 39.7 \mathrm{~ns}$ & $27.3 \pm 25.8$ & $63.5 \pm 29.5$ & $76.1 \pm 57.3$ & $59.2 \pm 31.3$ \\
\hline $\mathrm{C} 3 \mathrm{G}$ & $694.6 \pm 457.3 b$ & $177.4 \pm 198.8 \mathrm{a}$ & $223.3 \pm 171.7 \mathrm{ab}$ & $431.1 \pm 307.4 \mathrm{ab}$ & $227.5 \pm 218.6 \mathrm{ab}$ \\
\hline Pt3G & $14.7 \pm 11.1 \mathrm{~ns}$ & $2.9 \pm 2.5$ & $12.7 \pm 8.1$ & $14.9 \pm 12.2$ & $9.8 \pm 6.7$ \\
\hline TAC & $447.2 \pm 265.2 \mathrm{ab}$ & $152 \pm 127.8 \mathrm{a}$ & $542.8 \pm 264.9 b$ & $520.5 \pm 404.7 b$ & $291.3 \pm 237.7 \mathrm{ab}$ \\
\hline DPPH (IC50) & $46.3 \pm 17.5 \mathrm{~ns}$ & $64.9 \pm 14.5$ & $57.1 \pm 18.9$ & $59.4 \pm 15.2$ & $63.1 \pm 10.3$ \\
\hline ABTS (IC50) & $69.6 \pm 16.5 \mathrm{ab}$ & $80.9 \pm 22.7 b c$ & $60.4 \pm 4.1 \mathrm{a}$ & $87.6 \pm 20.3 c$ & $64.7 \pm 15.3 \mathrm{ab}$ \\
\hline FRAP (mg ASC/g) & $20.9 \pm 10.8 b$ & $10.8 \pm 5.3 \mathrm{a}$ & $21.6 \pm 3.0 \mathrm{~b}$ & $11.6 \pm 4.7 \mathrm{a}$ & $17.4 \pm 6.5 b$ \\
\hline TPC (mg GAE/g) & $25.0 \pm 5.0 \mathrm{~b}$ & $14.9 \pm 3.4 \mathrm{a}$ & $25.3 \pm 5.3 b$ & $16.4 \pm 4.8 \mathrm{a}$ & $22.7 \pm 6.6 b$ \\
\hline RACI & $0.6 \pm 0.8 b$ & $-0.5 \pm 0.5 \mathrm{a}$ & $0.7 \pm 0.5 b$ & $-0.5 \pm 0.7 \mathrm{a}$ & $0.2 \pm 0.7 b$ \\
\hline
\end{tabular}

Values within a column with different letters were statistically significant $(P<0.05$; Duncan's multiple range test $)$.

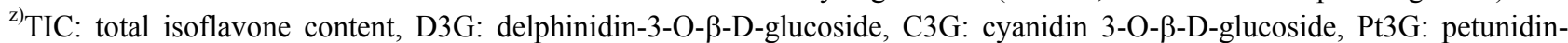
3-O- $\beta$-D-glucoside, TAC: total anthocyanin content, DPPH: 2,2-diphenyl-1-picrylhydrazyl, ABTS: 2,2'-azino-bis(3ethylbenzothiazoline-6-sulphonic acid), FRAP: ferric-reducing antioxidant power, ASC: ascorbic acid, TPC: total polyphenol content, GAE: gallic acid equivalents, RACI: relative antioxidant capacity index, ns: not significant.

Korean black soybean landraces had different rankings in antioxidant capacity (Supplementary Table 1). To compare data obtained by different chemical methods used to evaluate extract antioxidant activity, we used RACI for the 56 Korean black soybean landraces (Fig. 1). RACI results could be used to select black soybeans with higher antioxidant activity. Sample IT156132 had the highest antioxidant activity among the 56 Korean black soybean landraces. Therefore, RACI can be used to develop new breeding materials.

The black pigmentation of black soybeans is due to the accumulation of anthocyanins in the epidermis pallsade layer of the seed coat (Todd and Vodkin 1993). Anthocyanins are the second most important phytochemicals in black soybean besides isoflavones (Zhang et al. 2011). As revealed in previous studies, anthocyanin contents in black soybeans ranged from $<1.0$ to $20.4 \mathrm{mg} / \mathrm{g}$ (Xu and Chang 2008a; 2008b). Anthocyanins in seed coats play important roles in the protection against oxidative damage (Ramarathnam et al. 1989). Among them, C3G was found to be the most abundant anthocyanin in black soybeans. C3G has been reported to possess strong antioxidant activity in antioxidant assays among three anthocyanins isolated from black beans (Tsuda et al. 1994). In our study, three anthocyanins (D3G, C3G, and Pt3G) were identified. A wide variation of TAC (from 19.8 to $1,420.4 \mathrm{mg} / 100 \mathrm{~g}$ ) was also confirmed in the 56 Korean black soybean landraces (Table 1). Among three detected anthocyanins, $\mathrm{C} 3 \mathrm{G}$ was the most abundant anthocyanin in the 56 Korean black soybean landraces. In correlation analysis, TAC showed a positive correlation with RACI, although D3G, C3G, and Pt3G failed to show significant correlation with antioxidant activities.

In soybeans, isoflavones are synthesized through the 


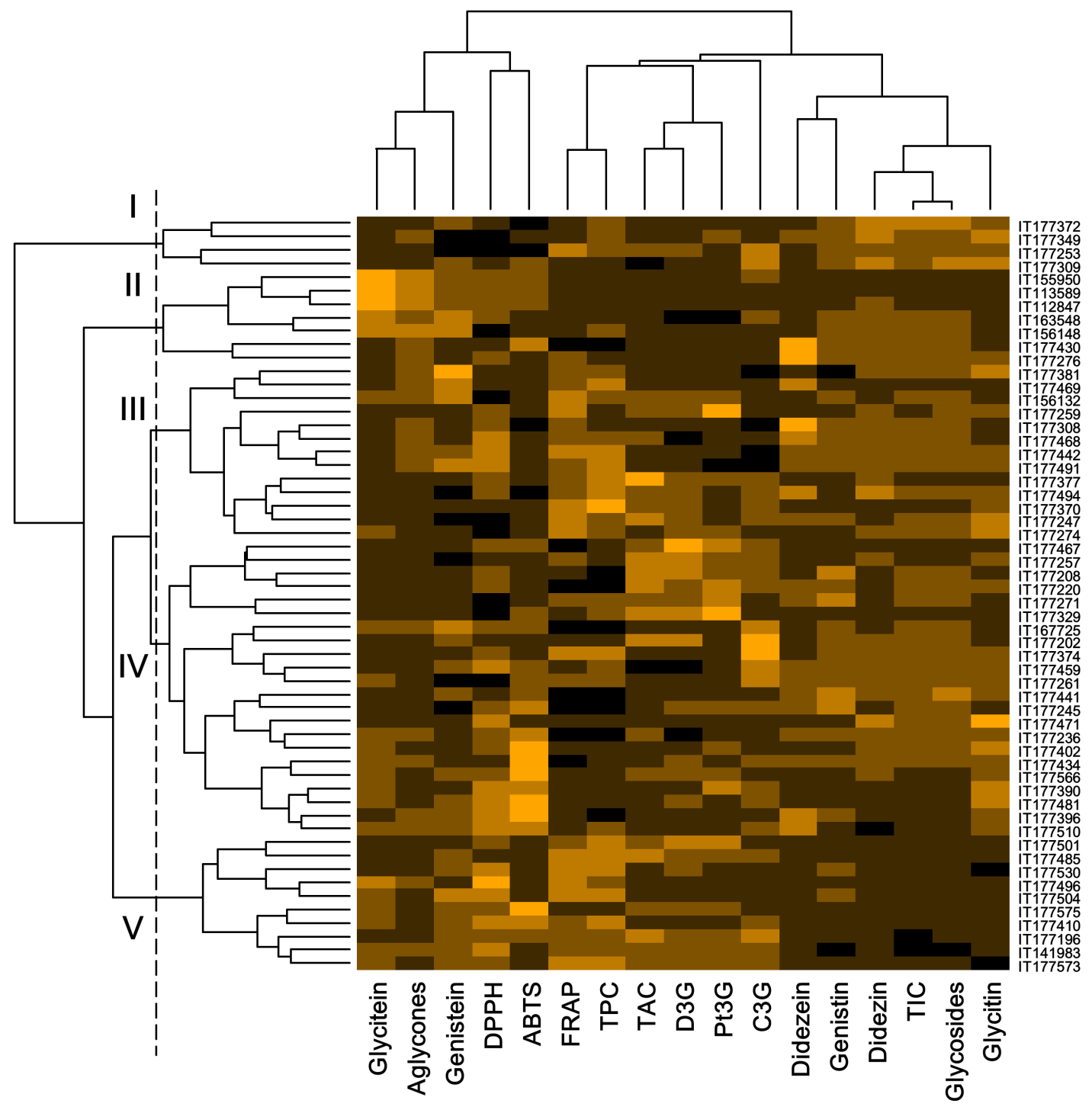

Fig. 3. Hierarchical clustering analysis of anthocyanins, isolavones, and antioxidant activities of the 56 Korean black soybean accessions.

DPPH: 2,2-diphenyl-1-picrylhydrazyl, ABTS: 2,2'-azino-bis(3-ethylbenzothiazoline-6-sulphonic acid), FRAP: ferricreducing antioxidant power, TPC: total polyphenol content, TAC: total anthocyanin content, D3G: delphinidin-3-O$\beta$-D-glucoside, Pt3G: petunidin-3-O- $\beta$-D-glucoside, C3G: cyanidin 3-O- $\beta$-D-glucoside, TIC: Total isoflavone content.

phenylpropanoid pathway. They are stored in the vacuole as glucosyl- and malonyl-glucose conjugates (Graham 1991). However, their contents vary significantly. They can be affected by both genotype and environmental conditions (Dixon and Paiva 1995; Lee et al. 2003). Several researchers have considered isoflavones as major phenolic compounds with concentration in different soybean varieties ranging from 126.1 to $409.2 \mathrm{mg} / 100 \mathrm{~g}$ of soybeans (Wang and Murphy 1994; Carrao-Panizzil and
Kitamura 1995; Tsukamoto et al. 1995). The 56 Korean black soybean landraces in this study showed various ranges of isoflavone contents, ranging from 43.8 to 347.5 $\mathrm{mg} / 100 \mathrm{~g}$ (Table 1), with glycitein contents ranging from 0.7 to $14.9 \mathrm{mg} / 100 \mathrm{~g}$. Some previous studies failed to detect glycitein in soybean seeds because it was below the limit of detection (Mujić et al. 2011; Sun et al. 2011). USDA has reported that glycitein content is ranging from 0.0 to 8.41 $\mathrm{mg} / 100 \mathrm{~g}$ in 49 soybean mature seeds (Bhagwat et al. 
2008). Glycitein synthesis is not yet clearly defined. It might have derived from isoliquiritigenin. In a human isoflavone metabolic study, a higher bioavailability of glycitein compared to that of genistein has been demonstrated (Latunde-Dada et al. 2001). Genistein could be metabolized into p-ethylphenol, which is not an estrogenic compound (Shutt et al. 1970). Song et al. (1999) have reported that glycitein has higher bioavailability than genistein in mice. They suggested that glycitein might have been metabolized into compounds with greater estrogenic potency than that of genistein (Song et al. 1999). Among 56 Korea black soybean landraces, two accessions showed higher glycitein contents (IT155950, 14.9 $\pm 1.8 \mathrm{mg} / 100 \mathrm{~g}$; IT113589, $14.4 \pm 1.2 \mathrm{mg} / 100 \mathrm{~g}$ ). The two soybean landraces may be used to understand glycitein biosynthesis so that a soybean variety that contains high isoflavone contents can be developed.

In summary, we analyzed anthocyanin and isoflavone contents of 56 Korean black soybean landraces in this study and estimated their antioxidant activities. In addition, we classified the 56 Korean black soybean landraces using PCA analysis and hierarchical clustering analysis. Our results could contribute to more efficient conservation and utilization of black soybean landraces to broaden the genetic bases of commercially grown varieties of soybean. Especially, IT177372, IT177349, IT177253, and IT177309 might be promoted as soy products in daily diet because they have higher contents of healthful factors.

\section{ACKNOWLEDGEMENTS}

This research was supported by the Research Program for Agricultural Science and Technology Development (Code no. PJ008623) funded by the National Academy of Agricultural Science, Rural Development Administration, Korea.

\section{REFERENCES}

Ajila CM, Prasada Rao UJ. 2008. Protection against hydrogen peroxide induced oxidative damage in rat erythrocytes by Mangifera indica L. peel extract. Food Chem. Toxicol.
46: 303-309.

Bellaloui N. 2012. Soybean seed phenol, lignin, and isoflavones and sugars composition altered by foliar boron application in soybean under water stress. Food Nutr. Sci. 3: 579-590.

Bhagwat SS, Haytowitz DB, Holden JM. 2008. USDA database for the isoflavone content of selected foods. U.S. Department of Agriculture, Beltsville, MD.

Brush SB. 1995. In-situ conservation of landraces in centers of crop diversity. Crop Sci. 35: 346-354.

Carrao-Panizzil MC, Kitamura K. 1995. Isoflavone content in Brazilian soybean cultivars. Breed. Sci. 45: 295-300.

Cho GT, Lee JR, Moon JK, Yoon MS, Baek HJ, Kang JH, et al. 2008. Genetic diversity and population structure of Korean soybean landrace [Glycine $\max$ (L.) Merr.]. J. Crop Sci. Biothech. 11: 83-90.

Choung MG, Baek IY, Kang ST, Han WY, Shin DC, Moon HP, et al. 2001. Isolation and determination of anthocyanins in seed coats of black soybean (Glycine $\max ($ L.) Merr.). J. Agr. Food Chem. 49: 5848-5851.

Correa CR, Li L, Aldini G, Carini M, Oliver Chen CY, Chun HK, et al. 2010. Composition and stability of phytochemicals in five varieties of black soybeans (Glycine max). Food Chem. 123: 1176-1184.

Dajanta K, Janpum P, Leksing W. 2013. Antioxidant capacities, total phenolics and flavonoids in black and yellow soybeans fermented by Bacillus subtilis: A comparative study of Thai fermented soybeans (thua nao). Int. Food Res. J. 20: 3125-3132.

Dixon RA, Paiva NL. 1995. Stress-induced phenylpropanoid metabolism. Plant Cell. 7: 1085-1097.

Dudonné S, Vitrac X, Coutière P, Woillez M, Mérillon JM. 2009. Comparative study of antioxidant properties and total phenolic content of 30 plant extracts of industrial interest using DPPH, ABTS, FRAP, SOD, and ORAC assays. J. Agr. Food Chem. 57: 1768-1774.

Graham TL. 1991. Flavonoid and isoflavonoid distribution in developing soybean seedling tissues and in seed and root exudates. Plant Physiol. 95: 594-603.

Hammer O, Harper DAT, Ryan PD. 2001. PAST: Paleontological statistics software package for education and data analysis. Paleontol. Electron. 4: 1-9.

Jarvis DI, Brown AH, Cuong PH, Collado-Panduro L, Latournerie-Moreno L, Gyawali S, et al. 2008. A global perspective of the richness and evenness of traditional 
crop-variety diversity maintained by farming communities. Proc. Natl. Acad. Sci. U.S.A. 105: 5326-5331.

Kim EH, Kim SL, Kim SH, Chung IM. 2012. Comparison of isoflavones and anthocyanins in soybean [Glycine max (L.) Merrill] seeds of different planting dates. J. Agr. Food Chem. 60: 10196-10202.

Kwon SH, Kim JR, Song HS, Im KH. 1974. Characteristics of important agronomic traits of Korean local soybean collections. Korean J. Breed. Sci. 6: 67-70.

Latunde-Dada AO, Cabello-Hurtado F, Czittrich N, Didierjean L, Schopfer C, Hertkorn N, et al. 2001. Flavonoid 6-hydroxylase from soybean (Glycine max L.), a novel plant P-450 monooxygenase. J. Biol. Chem. 276: 1688-1695.

Lee DJ, Lee JY. 2004. Antioxidant activity by DPPH assay. Korean J. Crop Sci. 49: 187-194.

Lee S, Lee J. 2009. Effects of oven-drying, roasting, and explosive puffing process on isoflavone distributions in soybeans. Food Chem. 112: 316-320.

Lee SJ, Yan WK, Ahn JK, Chung IM. 2003. Effects of year, site, genotype and their interactions on various soybean isoflavones. Field Crop Res. 81: 181-192.

Lee YH. 2003. Uses of Korean landrace soybean. Res. Bull. Korean Acad. Nat. Species 8: 15-36.

Mossi AJ, Cansian RL, Carvalho AZ, Dariva C, Oliveira JV, Mazutti M, et al. 2004. Extraction and characterization of volatile compounds in Maytenus ilicifolia, using highpressure CO2. Fitoterapia 75: 168-178.

Mujić I, Šertović E, Jokić S, Sarić Z, Alibabić V, Vidović S, et al. 2011. Isoflavone content and antioxidant properties of soybean seeds. Croat. J. Food Sci. Technol. 3: 16-20.

Nordberg J, Arnér ES. 2001. Reactive oxygen species, antioxidants, and the mammalian thioredoxin system. Free Radic. Biol. Med. 31: 1287-1312.

Perry MC, Mclntosh MS. 1991. Geographical patterns of variation in the USDA soybean germplasm collection. I. morphological traits. Crop Sci. 31: 1350-1355.

Ramarathnam N, Osawa T, Namiki M, Kawakishi S. 1989. Chemical studies on novel rice hull antioxidants. 2. Identification of isovitexin, a C-glycosyl flavonoid. J. Agric. Food Chem. 37: 316-319.

Re R, Pellegrini N, Proteggente A, Pannala A, Yang M, Rice-Evans C. 1999. Antioxidant activity applying an improved ABTS radical cation decolorization assay. Free Radic. Biol. Med. 26: 1231-1237.
Shin SC, Lee SJ, Lee SJ, Chung JI, Bae DW, Kim ST, et al. 2009. Comparison of anthocyanin content in seed coats of black soybean [Glycine max (L.) Merr.] cultivars using liquid chromatography coupled to tandem mass spectrometry. Food Sci. Biotechnol. 18: 1470-1475.

Shutt DA, Weston RH, Hogan JP. 1970. Quantitative aspects of phytoestrogen metabolism in sheep fed on subtreeanean clover (trifolium subterraneum cultivar clare) or red clover (trifolium pratense). Aust. J. Agr. Res. 21: 713-722.

Song HS, Lee YI, Kwon SH. 1991. Study on the agronomic traits of Korean native soybean (Glycine max). Korean Soybean Dig. 8: 1-16.

Song TT, Hendrich S, Murphy PA. 1999. Estrogenic activity of glycitein, a soy isoflavone. J. Agr. Food Chem. 47: 1607-1610.

Sthapit BR, Rao VR. 2009. Consolidating community's role in local crop development by promoting farmer innovation to maximise the use of local crop diversity for the well-being of people. International Society for Horticultural Science (ISHS), Leuven, Belgium.

Sun JM, Sun BL, Han FX, Yan SR, Yang H, Kikuchi A. 2011. Rapid HPLC method for determination of 12 isoflavone components in soybean seeds. Agr. Sci. China 10: 70-77.

Sun T, Tanumihardjo SA. 2007. An integrated approach to evaluate food antioxidant capacity. J. Food Sci. 72: R159-R165.

Todd JJ, Vodkin LO. 1993. Pigmented soybean (Glycine max) seed coats accumulate proanthocyanidins during development. Plant Physiol. 102: 663-670.

Tsuda T, Ohshima K, Kawakishi S, Osawa T. 1994. Antioxidative pigments isolated from the seeds of Phaseolus-vulgaris L. J. Agr. Food Chem. 42: 248-251.

Tsukamoto C, Shimada S, Igita K, Kudou S, Kokubun M, Okubo K, et al. 1995. Factors affecting isoflavone content in soybean seeds: Changes in isoflavones, saponins, and composition of fatty acids at different temperatures during seed development. J. Agr. Food Chem. 43: 1184-1192.

Wang HJ, Murphy PA. 1994. Isoflavone composition of American and Japanese soybeans in Iowa: Effects of variety, crop year, and location. J. Agr. Food Chem. 42: 1674-1677.

Waterhouse AL. 2003. Determination of total phenolics. In: R.E. Wrolstad, T.E. Acree, H. An, E.A. Decker, M.H. 
Penner, D.S. Reid, et al. (ed.). Current protocols in food analytical chemistry. John Wiley \& Sons, Inc., New York, NY.

Willcox JK, Ash SL, Catignani GL. 2004. Antioxidants and prevention of chronic disease. Crit. Rev. Food Sci. 44: 275-295.

Xu B, Chang SK. 2008a. Antioxidant capacity of seed coat, dehulled bean, and whole black soybeans in relation to their distributions of total phenolics, phenolic acids, anthocyanins, and isoflavones. J. Agr. Food Chem. 56: 8365-8373.

$\mathrm{Xu}$ B, Chang SK. 2008b. Characterization of phenolic substances and antioxidant properties of food soybeans grown in the North Dakota-Minnesota region. J. Agr. Food Chem. 56: 9102-9113.
Yao LH, Jiang YM, Shi J, Tomás-Barberán FA, Datta N, Singanusong R, et al. 2004. Flavonoids in food and their health benefits. Plant Foods Hum. Nutr. 59: 113-122.

Yen GC, Duh PD. 1993. Antioxidative properties of methanolic extracts from peanut hulls. J. Am. Oil Chem. Soc. 70: 383-386.

Yoon MS, Baek HJ, Lee JR, Kim HH, Cho YH, Ahn JW, et al. 2003. The major morphological characteristics and variations of soybean landraces. Korean J. Intl. Agri. 15: 294-303.

Zhang RF, Zhang FX, Zhang MW, Wei ZC, Yang CY, Zhang $\mathrm{Y}$, et al. 2011. Phenolic composition and antioxidant activity in seed coats of 60 Chinese black soybean (Glycine max L. Merr.) varieties. J. Agr. Food Chem. 59: 5935-5944. 